\title{
Endoscopic Detection and Surgical Repair of Congenital Tracheo-Esophageal-Fistula (TEF) \pm Esophageal Atresia (EA)
}

\author{
Ralf-Bodo Tröbs ${ }^{1 *}$, Werner Finke ${ }^{2}$ \\ ${ }^{1}$ Department of Pediatric Surgery, Marienhospital Herne, St. Elisabeth Group, Ruhr-University of Bochum, \\ Herne, Germany \\ ${ }^{2}$ Department of Anesthesiology and Surgical Intensive Care, Marienhospital Herne, St. \\ Elisabeth Group, Ruhr-University of Bochum, Herne, Germany \\ Email: *ralf-bodo.troebs@marienhospital-herne.de, werner.finke@marienhospital-herne.de
}

Received 13 September 2014; revised 10 October 2014; accepted 5 November 2014

Academic Editor: Carl E. Hunt, George Washington University School of Medicine and Health Sciences, USA

Copyright (C) 2014 by authors and Scientific Research Publishing Inc.

This work is licensed under the Creative Commons Attribution International License (CC BY).

http://creativecommons.org/licenses/by/4.0/

c) (i) Open Access

\begin{abstract}
Purpose: This study was performed to evaluate the management of tracheoesophageal fistula (TEF) \pm esophageal atresia (EA) under the guidance of preoperative tracheo-bronchoscopy (TrSc). Methods: Between 2007 and July 2014, a total of 26 consecutive newborns who underwent rigid TrSc for suspected TEF were identified. All associated charts and operation reports were retrospectively analyzed. Results: Distal TEF with EA (Gross C) predominated $(n=18)$. Furthermore, we managed 2 infants with proximal and distal TEF (Gross D) and 4 infants with isolated TEF (Gross E). In our hands, TrSc was feasible in infants with a birth weight above $1300 \mathrm{~g}$. Twenty-five fistulas were identified by endoscopy in 23 patients. In one infant with a birth weight below $1000 \mathrm{~g}$, an attempt to perform TrSc was interrupted, and urgent TEF closure was required. Fistula site at the carina was associated with a high rate of esophageal anastomosis under tension. During surgery, proximal TEF and isolated TEF were safely approached via right cervicotomy $(n=5)$. Conclusion: This study supports the routine use of rigid TrSc at the time of surgery. Rigid TrSc allowed the surgical team to identify the number and location of TEFs, and the incidence of side effects was low.
\end{abstract}

\section{Keywords}

Tracheoesophageal Fistula, Esophageal Atresia, Tracheo-Bronchoscopy, Airway Management, Cervical Approach

\footnotetext{
"Corresponding author.
}

How to cite this paper: Tröbs, R.-B. and Finke, W. (2014) Endoscopic Detection and Surgical Repair of Congenital TracheoEsophageal-Fistula (TEF) \pm Esophageal Atresia (EA). Open Journal of Pediatrics, 4, 283-290. 


\section{Introduction}

The overall prevalence of esophageal atresia (EA) in Europe is 2.43 cases per 10,000 births; locally, the prevalence can be as high as 4.5 per 10,000 births (Mainz, Germany) [1]. In more than 4 of every 5 cases, EA is associated with at least one tracheoesophageal fistula (TEF). The typical symptoms of TEF include excessive salivation, coughing, choking, and apneic or cyanotic attacks soon after birth [2]. Failure to pass a nasogastric tube into the stomach and the presence of air in the upper gastrointestinal tract on X-ray of the thorax and abdomen indicate the presence of distal TEF with EA. At surgery closure of TEF is the most important step to stabilize the infant and to prevent severe airway morbidity.

In our institution, preoperative tracheo-bronchoscopy (TrSc) is routinely employed to determine the presence, site, and number of TEFs. However, according to a recent international survey on the management of esophageal atresia, the use of perioperative endoscopy remains controversial [3] [4]. The aim of the present study was to evaluate the primary management of TEF \pm EA in a medium-sized center with 2200 operations per year. TrSc and surgical repair were routinely performed within the same operation. As a follow-up, we assessed surgically relevant airway morbidity over the first 3 months postoperatively.

\section{Material and Methods}

Between 2006 and July 2013, TrSc for suspected congenital TEF was performed in 26 consecutive newborn infants. We retrospectively collected data by reviewing all records and operation protocols. We extracted the following data from the records: birth weight, gender, age at operation, results of TrSc, side effects, surgical management of TEF, and events during follow-up. The data are expressed as medians and ranges; further, the arithmetic mean and standard deviation were estimated.

In 17 cases with distal TEF + EA the site of TEF was estimated endoscopically. According to the distance from the carina infants were divided into 2 groups: 1) fistula site very close to or at the carina (group 1); 2) fistula located more proximal at the membranous part of the trachea (group 2).

Technique of tracheo-bronchoscopy

TrSc was always performed by the same team, consisting of a pediatric surgeon in close cooperation with a pediatric anesthesiologist. The infant was placed in a supine position. Endoscopy was started after the induction of anesthesia with thiopentone and fentanyl. After spraying the glottis and trachea with lignocaine, a rigid bronchoscope of an appropriate diameter (Karl Storz ${ }^{\circledR}$, Tuttlingen, Germany; Nos. 2.5 and 3.0; outer diameters 4 and $5 \mathrm{~mm}$, respectively) with an angulated rod-lens was inserted, and a T-piece breathing system (Mapleson D, Intersurgical Ltd. ${ }^{\circledR}$, Wokingham, Berkshire, UK) was attached to the side arm of the bronchoscope to continue manual ventilation. Monitoring included electrocardiogram, pulse oximetry, noninvasive blood pressure measuring, and estimation of the breathing rate and pressure. After introducing the instrument, the larynx, vocal folds, and posterior membranous trachea were carefully examined. After $\mathrm{TrSc}$, surgical repair was immediately continued.

\section{Results}

We identified 18 male and 8 female infants with a median BW of $2770 \mathrm{~g}(2575 \pm 1100)$. The smallest infant weighed $983 \mathrm{~g}$, and the maximum infant BW was $3600 \mathrm{~g}$. The distribution of body weights is presented in Figure 1. The different types of TEF included in this study are depicted in Figure 2. Surgical repair always began with endoscopy of the airway, and surgery was immediately continued with dissection of the TEF and esophageal anastomosis, if required. With a single exception, the infants with EA underwent operations within the first 3 days of life (1 delayed presentation underwent operation on day 9) [5]. The median age at operation for TEF + EA was 1 day. In contrast, infants with isolated TEF underwent operation on postnatal days 5, 6, and 8. One infant who underwent operation on postnatal day 55 was diagnosed with trisomy 18 and remained in a stable condition over the first month. In two infants suspected of having TEF, the presence of TEF was excluded endoscopically. In one of these infants, polyhydramnios, absent stomach, and a mediastinal abnormality were detected prenatally with ultrasound, and severe postnatal impairment of swallowing was obvious. From these symptoms, a definitive diagnosis of CHARGE syndrome associated with paraesophageal hernia was made. In the second case, the presence of pure EA (Gross A) without proximal fistula was confirmed by the exclusion of an upper pouch fistula. No infants exhibited proximal TEF with EA (Gross B). In 18 infants, EA with a single distal fis- 


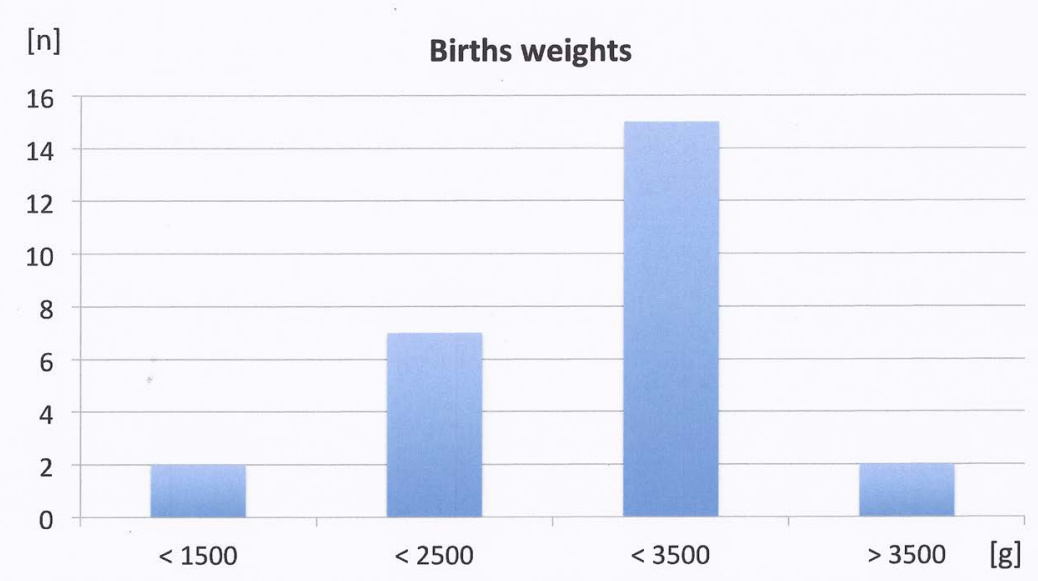

Figure 1. Distribution of birth weights.

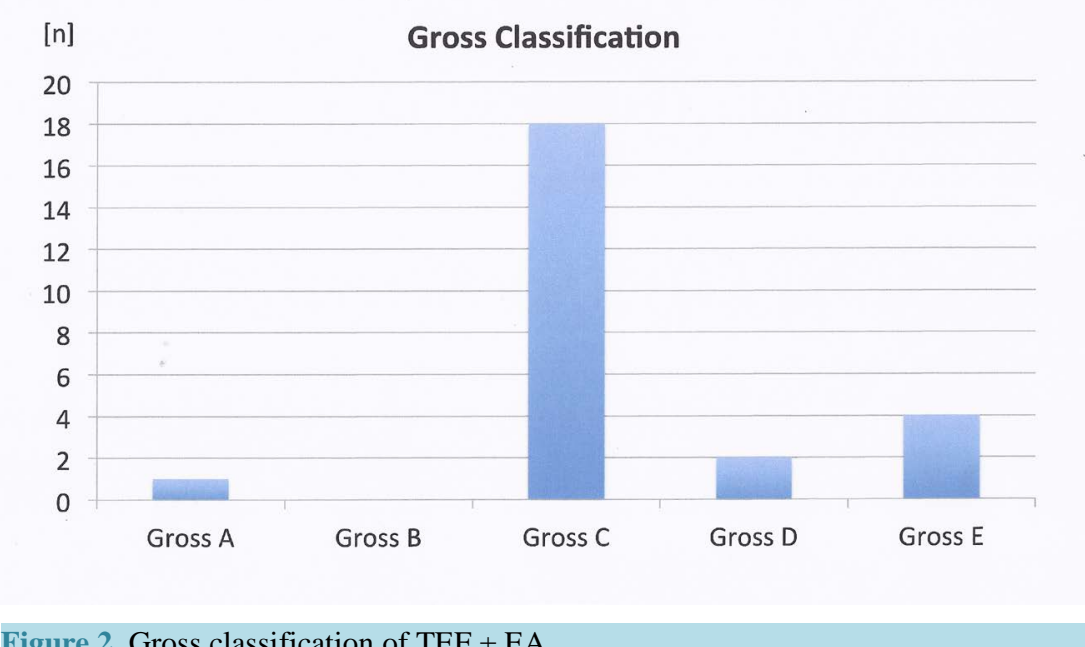

Figure 2. Gross classification of TEF \pm EA.

tula (Gross C) was diagnosed. In the smallest infant of this series (BW $983 \mathrm{~g}$ ), TrSc was attempted but could not be completed due to respiratory instability and the disproportionate size of the instrument and the entry to the larynx. In this patient, thoracotomy was immediately performed to dissect the TEF and to create a temporary gastrostomy. Esophageal anastomosis was established in a second operation conducted after weight gain.

In 2 infants in the EA group with air-filled stomachs on X-ray, TrSc revealed the presence of an additional proximal upper pouch fistula (Gross D). Dissection and closure of both fistulas were performed in one case exclusively via thoracotomy, and in the $2^{\text {nd }}$ case via additional right cervicotomy. In 4 patients, an isolated TEF (Gross E) was identified. For repair of isolated TEFs, we employed the right cervical approach in all cases ( $\mathrm{n}=$ 4).

\subsection{Fistula Site and Type of Anastomosis}

In 9 cases the TEF was found at the carina (group 1), and in 8 cases TrSc revealed a more cephalad position (group 2). In 6 cases of group 1 esophageal anastomosis was established using full thickness flap from the upper pouch (3 cases conventional anastomosis). In the total of infants of group 2 esophageal anastomosis was established conventionally.

The spectrum of different types of TEF is shown in the Figures 3-5. 


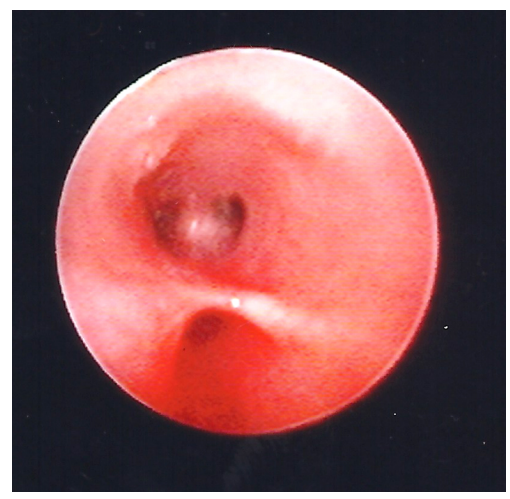

Figure 3. TEF distant from the carina (Gross C; group 2).

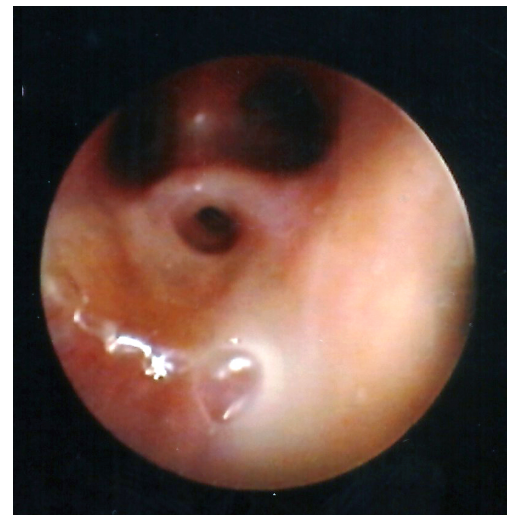

Figure 4. "Trifurcation" due to TEF just above the carina (Gross C; group 1).

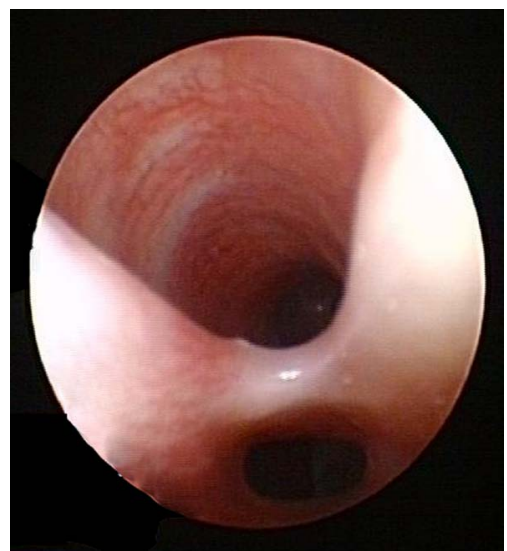

Figure 5. Isolated cervical TEF (Gross E).

\subsection{Additional Findings and Follow-Up}

Additional endoscopic findings of TRSC included the tracheal origin of the right upper lobe bronchus $(n=1)$, and gradual tracheal narrowing $(\mathrm{n}=1)$. In this series, we did not observe any perioperative mortality. One infant died 2 months postoperatively due to pulmonary hypertension of unknown origin. Severe tracheomalacia occurred in 2 infants with TEF Gross C and D, respectively. In one case, aorto-truncopexy improved symptom, but in the second child with trisomy 21, tracheostomy was required due to subglottic stenosis and extended tracheomalacia. During a median follow up of 3.5 years (range: 3 months to 7 years), relapsed TEF was not observed in any of the cases. 


\subsection{Limitations}

The presented study is limited by retrospective character. It includes a relative small number of patients, and a comparison group is lacking. Data are interpreted on the background of the literature. Furthermore, classification of the fistula site was extrapolated retrospectively from the operation records and photographs. Both authors were involved directly and in a responsible position in the treatment of the total of patients.

\section{Discussion}

\subsection{Tracheobronchoscopy}

Our data support the routine use of TrSc to confirm the presence and location of proximal and distal TEFs at the time of surgical repair. This investigation is performed in the operative theater, and surgical correction was undertaken immediately after endoscopy. TrSc was feasible even in infants with BWs above $1300 \mathrm{~g}$. The most important benefit of TrSc seems to be the identification of unrecognized proximal upper pouch fistulas. Even in infants with gasless abdomens upon X-ray analysis, upper pouch fistulas may nonetheless be present. Missed proximal TEFs at the primary surgical repair will always become symptomatic, with both respiratory symptoms and recurrent pneumonias. In a previous pioneering study, endoscopic findings influenced the operative procedure in more than 57 patients [6]. In a recent large multicenter study, the reported rate of repeated TEF repairs was 5.4\% within 2 years; however later recurrences may still occur [7] [8]. This statistic includes relapses due to recanalization of the original fistula as well as missed proximal TEFs after the repair of Gross type C atresia. A tertiary center study described the predominance of missed proximal TEFs in the case of reoperation [9]. In an extensive meta-analysis, the incidence of isolated proximal fistula (Gross type B) was 1.14\%, and combined proximal and distal fistulas (Gross type D) occurred in $0.98 \%$ of infants. After the introduction of routinely performed $\mathrm{TrSc}$ at this institution, the incidence of proximal TEF increased significantly [10]. In another series TEFs were identified with TrSc in a cohort of infants with suspected pure EA [11].

However, even with the use of TrSc, a proximal fistula can be overlooked in rare instances [12]. Even after extensive mobilization of the proximal pouch, a proximal TEF may be missed because these upper pouch fistulas are rarely located at the end of the proximal esophagus [10] [12]. In 2 of our patients with upper pouch TEFs, crucial findings with TrSc led to a change in the operative procedure.

Another advantage of TrSc is that the level of an isolated/proximal TEF can be estimated either by transillumination or digitally by palpating the tip of the instrument.

Furthermore, the working channel of rigid tracheoscopes allows the introduction of a 2- to 3-F catheter into the fistula. This maneuver can help to decompress the stomach [11]. In addition, insufflation of the balloon with a Fogarty catheter allows temporary occlusion of the TEF. This technique was introduced to stabilize the preoperatively unstable infant with respiratory distress on the ICU and as the initial step in airway management at surgery as well [13]-[16]. However, balloon occlusion was not very successful in our hands because the catheter slipped out —an experience that has been reported by others as well [2].

As an alternative to rigid endoscopes, flexible ultrathin fiberscopes have been developed that allow endoscopy through the endotracheal tube [17]. This type of instrument might be advantageous, especially for intraoperative use, as it allows for the tracheoscopically assisted ligation and dissection of TEFs [18] [19].

As an alternative to TrSc, contrast medium ingestion or pull-back tube fluorography (PBF) with a water-soluble medium can be employed as a diagnostic tool for the detection of proximal TEF in EA. However, the latter procedure requires “a high degree of radiologic pediatric expertise” [10]. Furthermore, the procedure may be associated with an enhanced risk of aspiration, and over-interpretation may occur. In cases of EA, false positive results may occur due to an overflow of contrast medium into the tracheobronchial tree [20]. Furthermore, mucous occlusion of the fistula may lead to false negative results. In the presence of isolated TEF without EA, the anatomy allows for successful radiological visualization of the membranous trachea. The fistula runs in an oblique direction upward from its origin in the esophagus; due to its anatomy, this type of fistula is more precisely described as an "N-type fistula" [21]. In one of our cases, PBF was performed successfully prior to admission to our department.

TrSc has the potential to cause severe complications, including oxygen desaturation, laryngospasm, bleeding, pneumothorax, and gastric overextension [22]-[24]. No patient in our series developed pneumothorax or gastrointestinal rupture immediately after TrSc. However, in the smallest patient in our group, an attempt to perform TrSc was terminated because of ventilation difficulty. Intraoperatively, this patient developed a right-sided 
tension pneumothorax, and pleural drainage was required. In a second case with an isolated H-fistula, high airflow through the TEF occurred during facemask ventilation, and urgent closure of the TEF was performed via extrapleural cervicotomy, leading to stabilization. Postoperatively, this patient presented with right pneumothorax. This pneumothorax disappeared spontaneously under low-pressure ventilation.

In the presented series, rigid TrSc enabled the identification of both the location and number of TEFs in all cases investigated.

To minimize the time for endoscopy, we mainly focused our attention on the detection of TEF following insertion of the endoscope. In two infants, additional anomalies of the tracheobronchial tree were registered (ectopic right upper bronchus and gradual narrowing of trachea); both cases were resolved were without further surgical consequences [17]. This rate is much lower than reported previously by others [25]. Generally, a high rate of primary and secondary tracheobronchial problems is associated with EA + TF and can be recognized with a sophisticated technique and repeated investigations.

In $1 \%$ of infants, CHARGE syndrome is associated with EA \pm TEF [1] [17]. Our patient presented with prenatal signs and postnatal symptoms of EA ("bubble blowing neonate"); however, both were excluded by TrSc [1] [26].

In the latest review paper, Parolioni et al. (2014) came to the conclusion that TrSc with a rigid tracheoscope "has proven most useful in the diagnostic and therapeutic assessment of newborns" with EA [27].

\subsection{Surgery for TEF}

The standard surgical approach for the repair of distal TEF is the muscle-saving posterolateral right thoracotomy with extrapleural preparation or thoracoscopic repair [2] [3]. A multicenter overview has reported that thoracoscopic repair of EA \pm TEF is still performed only at select centers [4].

In two thirds of our patients with TEF found at the carina (group 1) we used an upper pouch muco-muscular flap to bridge a wide gap EA [28] [29]. In contrast, no patient of group 2 required upper pouch flap technique. Thus, our results support the observation, that the presence of carinal TEF is associated with wide gap EA [6].

In our series, isolated TEFs were safely dissected and closed via right cervicotomy [30] [31]. In selected cases of EA in which the distal TEF is located above the clavicle, successful cervical repair has been reported [32]. In cervical repair, dissection is performed anterior to the sternocleidomastoid, and exposure of the trachea-esophageal complex into the upper mediastinum is required. Loupe magnification is strongly recommended to preserve the recurrent laryngeal (RLN) nerve [30] [33]. None of our patients developed signs of RLN palsy. However, unilateral RLN palsy may occur in a number of children after EA repair and may be nearly asymptomatic [34]. Additionally, vocal fold paralysis with serious consequences, including tracheostomy, can occur [35] [36]. For the approach to the pharyngeal esophagus, it must be noted that extensive mobilization should be avoided to preserve the multiple tiny motor branches of the RLN to the pharyngeal esophagus [12] [37] [38]. With the introduction of thoracoscopic repair for isolated fistulas in neonates and older children, the paradigm of open surgery for isolated TEFs may be questioned [39] [40].

\section{Conclusion}

In conclusion, rigid TrSc is a very useful tool for TEF identification. The use of the technique is limited in very unstable infants and in infants with extremely low birth weights. The cervical surgical approach to proximal/isolated TEF exhibits some advantages. Applying this approach, the surgeon should keep in mind that the recurrent laryngeal nerve poses a relevant surgical risk.

\section{Disclosure Statement}

The authors have no competing financial interests to declare.

\section{Ethics Statement}

This study was approved by the ethics committee of the Ruhr-University of Bochum.

\section{References}

[1] Pedersen, R.N., Calzolari, E., Husby, S. and Garne, E., EROCAT Working Group (2012) Oesophageal Atresia: Preva- 
lence, Prenatal Diagnosis and Associated Anomalies in 23 European Regions. Archives of Disease in Childhood, 97, 227-232. http://dx.doi.org/10.1136/archdischild-2011-300597

[2] Pinheiro, P.F.M., Simoes e Silva, A.C. and Pereira, R.M. (2012) Current Knowledge on Esophageal Atresia. World Journal of Gastroenterology, 18, 3662-3672. http://dx.doi.org/10.3748/wjg.v18.i28.3662

[3] Spitz, L. (2007) Oesophageal atresia. Orphanet Journal of Rare Diseases, 2, 24. http://dx.doi.org/10.1186/1750-1172-2-24

[4] Zani, A., Eaton, S., Hoellwarth, M.E., Puri, P., Tovar, J., Fasching, G., Bagolan, P., Lukac, M., Wijnen, R., Kuebler, J.F., Cecchio, G., Rintala, R. and Pierro, A. (2014) International Survey on the Management of Esophageal Atresia. European Journal of Pediatric Surgery, 24, 3-8. http://dx.doi.org/10.1055/s-0033-1350058

[5] Tröbs, R.B. and Becker, J.C. (2014) Emergency out of Hospital Presentation of Neonatal Esophageal Atresia with Esophagotracheal Fistula. Klinische Pädiatrie, 214, 47-48.

[6] Kosloske, A.M., Jewell, P.F. and Cartwright, K.C. (1988) Crucial Bronchoscopic Findings in Esophageal Atresia and Tracheoesophageal Fistula. Journal of Pediatric Surgery, 23, 466-470. http://dx.doi.org/10.1016/S0022-3468(88)80450-0

[7] Sulkowski, J.P., Cooper, J.N., Lopez, J.J., Jadcherla, Y., Cuenot, A., Mattei, P., Deans, K.J. and Minneci, P.C. (2014) Morbidity and Mortality in Patients with Esophageal Atresia. Surgery, 156, 483-491. http://dx.doi.org/10.1016/j.surg.2014.03.016

[8] Tamay, Z., Güler, N., Kilic, G., Gün, F., Celik, A. and Yekeler, E. (2008) A Congenital Proximal Tracheoesophageal Fistula 14 Years after Surgical Repair of Esophageal Atresia with Distal Tracheoesophageal Fistula. The Turkish Journal of Pediatrics, 50, 299-301.

[9] Guo, W., Li, Y., Peng, Y., Huo, D. and Chen, Y. (2010) Tracheoesophageal Fistula after Primary Repair of Type C Esophageal Atresia in the Neonatal Period: Recurrent or Missed Second Congenital Fistula. Journal of Pediatric Surgery, 45, 2351-2355. http://dx.doi.org/10.1016/j.jpedsurg.2010.08.030

[10] Parolini, F., Morandi, A., Macchini, F., Canazza, L., Torricelli, M., Zanini, A. and Leva, E. (2013) Esophageal Atresia with Proximal Tracheaesophageal Fistula: A Missed Diagnosis. Journal of Pediatric Surgery, 48, E13-E17. http://dx.doi.org/10.1016/j.jpedsurg.2013.04.018

[11] Sharma, N. and Srinivas, M. (2014) Laryngotracheobronchoscopy Prior to Esophageal Atresia and Tracheoesophageal Fistula Repair-Its Use and Importance. Journal of Pediatric Surgery, 49, 367-369. http://dx.doi.org/10.1016/j.jpedsurg.2013.09.009

[12] Bax, K.M.A., Roskott, A.M. and van der Zee, D.C. (2008) Esophageal Atresia without Distal Tracheoesophageal Fistula: High Incidence of Proximal Fistula. Journal of Pediatric Surgery, 43, 522-525. http://dx.doi.org/10.1016/j.jpedsurg.2007.10.034

[13] Benjamin, B. (1981) Endoscopy in Esophageal Atresia and Tracheoesophageal Fistula. Annals of Otology, Rhinology \& Laryngology, 90, 376-382. http://dx.doi.org/10.1177/000348948109000418

[14] Filston, H.C., Chitwood, W.R., Schkolne, B. and Blackmon, L.R. (1982) The Fogarty Balloon Catheter as an Aid to Management of the Infant with Esophageal Atresia and Tracheoesophageal Fistula Complicated by Severe RDS or Pneumonia. Journal of Pediatric Surgery, 17, 149-151. http://dx.doi.org/10.1016/S0022-3468(82)80199-1

[15] Filston, H.C., Rankin, J.S. and Grimm, J.K. (1984) Prognostic Factors and Contribution of Preoperative Telescopic Endoscopy. Esophageal Atresia. Annals of Surgery, 199, 532-536. http://dx.doi.org/10.1097/00000658-198405000-00007

[16] Reeves, S.T., Burt, N. and Smith, C.D. (1995) Is It Time to Reevaluate the Airway Management of Tracheoesophageal Fistula? Anesthesia \& Analgesia, 81, 866-869.

[17] Usui, N., Kamata, S., Ishikawa, S., Sawai, T., Okuyama, H., Imura, K. and Okada, A. (1996) Anomalies of the Tracheobronchial Tree in Patients with Esophageal Atresia. Journal of Pediatric Surgery, 31, 258-262. http://dx.doi.org/10.1016/S0022-3468(96)90010-X

[18] Deanovic, D., Gerber, A.C., Meuli, M. and Weiss, M. (2007) Traceoscopy Assisted Repair of Tracheo-Esophageal Fistula (TARTEF): A 10-Year Experience. Pediatric Anesthesia, 17, 557-562. http://dx.doi.org/10.1111/j.1460-9592.2006.02147.x

[19] De Gabriele, L.C., Cooper, M.G., Singh, S. and Pitkin, J. (2001) Intraoperative Fibreoptic Bronchoscopy during Neonatal trachea-Oesophageal Fistula Ligation and Esophageal Atresia Repair. Anaesth Intensive Care, 29, 284-287.

[20] Gopal, M. and Woodward, M. (2007) Potential Hazards of Contrast Study Diagnosis of Esophageal Atresia. Journal of Pediatric Surgery, 42, e9-e10. http://dx.doi.org/10.1016/j.jpedsurg.2006.11.008

[21] Laffan, E.E., Daneman, A., Ein, S.H., Kerrigan, D. and Manson, D.E. (2006) Tracheoesophageal Fistula without Esophageal Atresia: Are Pull-Back Tube Esophagograms Needed for Diagnosis. Pediatric Radiology, 36, 1141-1147. 
http://dx.doi.org/10.1007/s00247-006-0269-0

[22] Iannoli, E.D. and Litman, R.S. (2002) Tension Pneumothorax during Flexible Fiberoptic Bronchoscopy in a Newborn. Anesthesia \& Analgesia, 94, 512-513. http://dx.doi.org/10.1097/00000539-200203000-00007

[23] Veyckemans, F., Hamoir, M., Rombeaux, P., van Obbergh, L.J. and Reding, R. (2002) Preoperative Tracheoscopy in Neonates with Esophageal Atresia. Letter to the Editor. Anesthesia \& Analgesia, 95, 1827-1828. http://dx.doi.org/10.1097/00000539-200212000-00088

[24] Knottenbelt, G., Skinner, A. and Seefelder, C. (2010) Tracheo-Oesophageal Fistula (TOF) and Oesophageal Atresia (OA). Best Practice \& Research Clinical Anaesthesiology, 24, 387-401. http://dx.doi.org/10.1016/j.bpa.2010.02.010

[25] Holzki, J. (1992) Bronchoscopic Findings and Treatment in Congenital Tracheo-Oesophageal Fistula. Pediatric Anesthesia, 2, 297-303. http://dx.doi.org/10.1111/j.1460-9592.1992.tb00220.x

[26] Kutiyanawala, M., Wyse, R.K.H., Brereton, J., Spitz, L., Kiely, E.M., Drake, D. and Blake, K. (1992) CHARGE and Esophageal Atresia. Journal of Pediatric Surgery, 27, 558-560. http://dx.doi.org/10.1016/0022-3468(92)90445-D

[27] Parolini, F., Boroni, G., Stefini, S., Agapiti, C., Bazzana, T. and Alberti, D. (2014) Role of Preoperative Tracheobronchoscopy in Newborns with Esophageal Atresia. World Journal of Gastrointestinal Endoscopy, 6, 482-487. http://dx.doi.org/10.4253/wjge.v6.i10.482

[28] Gough, M.H. (1980) Esophageal Atresia-Use of an Anterior Flap in the Difficult Anastomosis. Journal of Pediatric Surgery, 15, 310-311. http://dx.doi.org/10.1016/S0022-3468(80)80143-6

[29] Bar-Maor, J.A., Shoshany, G. and Sweed, Y. (1989) Wide Gap Esophageal Atresia: A New Method to Elongate the Upper Pouch. Journal of Pediatric Surgery, 24, 882-883. http://dx.doi.org/10.1016/S0022-3468(89)80587-1

[30] Riazulhaq, M. and Elhassan, E. (2012) Early Recognition of H-Type Tracheoesophageal Fistula. APSP Journal of Case Reports, 3, 4.

[31] Monnier, P. (2011) Pediatric Airway Surgery. Oesophageal Atresia with Tracheo-Oesophageal Fistula. Springer, Berlin.

[32] Kemmotsu, H., Joe, K., Nakamura, H. and Yamashita, M. (1995) Cervical Approach for the Repair of Esophageal Atresia. Journal of Pediatric Surgery, 30, 549-552. http://dx.doi.org/10.1016/0022-3468(95)90128-0

[33] Killen, D.A. and Greenlee, H.B. (1965) Transcervical Repair of H-Type Congenital Tracheo-Esophageal Fistula: Review of the Literature. Annals of Surgery, 162, 145-150. http://dx.doi.org/10.1097/00000658-196507000-00021

[34] Bargy, F., Manach, Y., Halardot, P.G. and Bienayme, J. (1983) Risk of Recurrent Laryngeal Nerve Palsy in Surgery of Esophageal Atresia. Chirugie Pediatrique, 24, 130-132.

[35] Oestreicher-Kedem, Y., DeRowe, A., Fishman, G. and Ben-Ari, J. (2008) Vocal Fold Paralysis in Infants with Tracheoesophageal Fistula. Annals of Otology, Rhinology \& Laryngology, 117, 896-901. http://dx.doi.org/10.1177/000348940811701206

[36] Mortellaro, V.E., Pettiford, J.N., St Peter, S.D., Fraser, J.D., Ho, B. and Wei, J. (2011) Incidence, Diagnosis, and Outcomes of Vocal Fold Immobility after Esophageal Atresia (EA) and/or Tracheoesophageal Fistula (TEF) Repair. European Journal of Pediatric Surgery, 21, 386-388. http://dx.doi.org/10.1055/s-0031-1291269

[37] Davies, M.R.Q. (1996) Anatomy of the Extrinsic Nerve Supply of the Oesophagus in Oesophageal Atresia of the Common Type. Pediatric Surgery International, 11, 230-233. http://dx.doi.org/10.1007/BF00178424

[38] Fragoso, A.C. and Tovar, J.A. (2014) The Multifactorial Origin of Respiratory Morbidity in Patients Surviving Neonatal Repair of Esophageal Atresia. Frontiers in Pediatrics, 2, 39.

[39] Aziz, G.A. and Schier, F. (2005) Thoracoscopic Ligation of a Tracheoesophageal H-Type Fistula in a Newborn. Journal of Pediatric Surgery, 40, e35-e36. http://dx.doi.org/10.1016/j.jpedsurg.2005.03.037

[40] Lisle, R.M., Nataraja, R.M. and Mahomed, A.A. (2010) Technical Aspects of the Thoracoscopic Repair of a Late Presenting Congenital H-Type Fistula. Pediatric Surgery International, 26, 1233-1236. http://dx.doi.org/10.1007/s00383-010-2678-2 
Scientific Research Publishing (SCIRP) is one of the largest Open Access journal publishers. It is currently publishing more than 200 open access, online, peer-reviewed journals covering a wide range of academic disciplines. SCIRP serves the worldwide academic communities and contributes to the progress and application of science with its publication.

Other selected journals from SCIRP are listed as below. Submit your manuscript to us via either submit@scirp.org or Online Submission Portal.
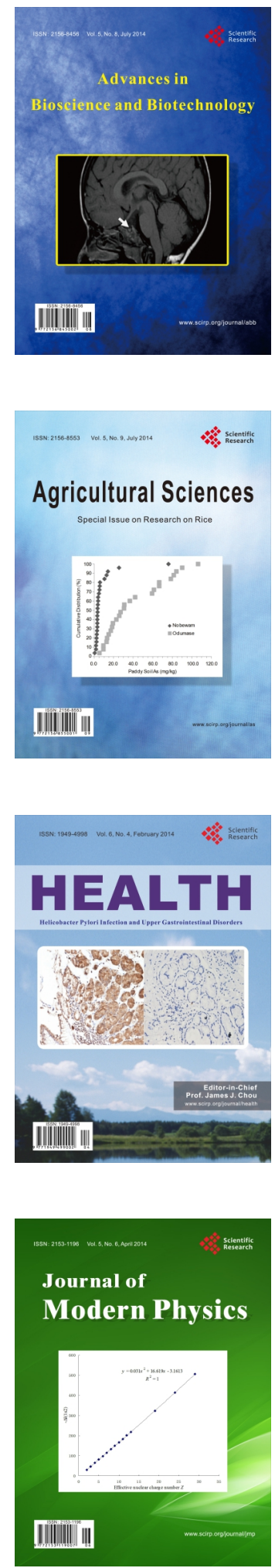
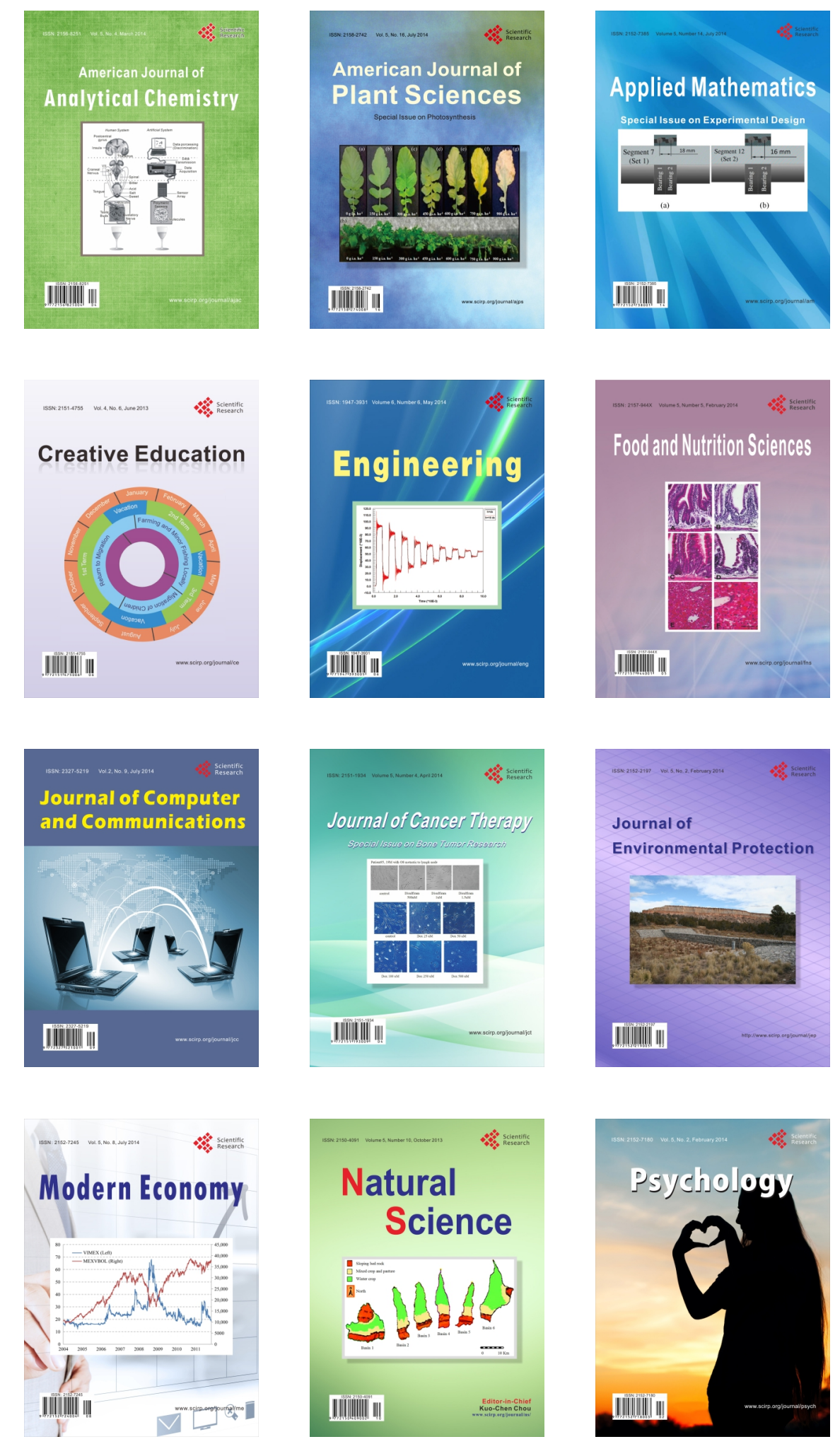